\section{EMBRYRIDDLE Aeronautical University}

SCHOLARLY COMMONS

\section{International Journal of Aviation,} Aeronautics, and Aerospace

\title{
Not-So-Risky Business? Assessing the Risk of Integrating Large RPVs into the Current Air Traffic System
}

\author{
Hans-Joachim K. Ruff-Stahl \\ Embry-Riddle Aeronautical University - Worldwide, ruffha@erau.edu \\ Steven Esser \\ Embry-Riddle Aeronautical University - Worldwide, david.esser@erau.edu \\ Dirk Farsch \\ Embry-Riddle Aeronautical University - Worldwide, farschd@my.erau.edu \\ Thomas Graner \\ Embry-Riddle Aeronautical University - Worldwide, granert@my.erau.edu \\ Robert Klein \\ Embry-Riddle Aeronautical University - Worldwide, kleinr6@my.erau.edu \\ Christian Schneidt \\ Embry-Riddle Aeronautical University - Worldwide, schneic4@my.erau.edu \\ Stefan Schoenborn \\ Embry-Riddle Aeronautical University - Worldwide, schonbos@my.erau.edu
}

Follow this and additional works at: https://commons.erau.edu/ijaaa

Part of the Risk Analysis Commons

\section{Scholarly Commons Citation}

Ruff-Stahl, H. K., Esser, S., Farsch, D., Graner, T., Klein, R., Schneidt, C., \& Schoenborn, S. (2016). Not-SoRisky Business? Assessing the Risk of Integrating Large RPVs into the Current Air Traffic System. International Journal of Aviation, Aeronautics, and Aerospace, 3(1). https://doi.org/10.15394/ ijaaa.2016.1103

This Article is brought to you for free and open access by the Journals at Scholarly Commons. It has been accepted for inclusion in International Journal of Aviation, Aeronautics, and Aerospace by an authorized administrator of Scholarly Commons. For more information, please contact commons@erau.edu. 
Over the last decade, governments and private sectors worldwide have put considerable effort into the commercialization of Remotely Piloted Vehicles (RPV) (also known as Unmanned Aircraft Systems [UAS], Unmanned Aerial Systems [UAS] or Unmanned Aerial Vehicles [UAV]). By 2015, the maturity of RPVs has reached an unprecedented level; foreseeable military and civil applications are ranging from surveillance and environmental observations to agricultural operations and cargo transport. While RPVs offer several distinct advantages over manned aircraft, their mode of operation still presents unsolved challenges. Yet despite their maturity and potential, an integration of RPVs into the existing Air Transportation System (ATS) and Airspace Structure is pending (BBC, 2013 and FAA, n.d.).

While the International Civil Aviation Organization (ICAO) has identified the need for technical solutions and a different regulatory framework for the safe integration into non-segregated airspace (ICAO, 2011), the goal of this study is to assess the risk of an integration of RPVs into the present state ATS on the basis of current regulations. It is argued that a Heron 1 type RPV may be safely integrated in European airspace within the existing airspace structure and governing rules, even without the availability of detect-and-avoid technology.

\section{The Heron 1 RPV System}

The Heron 1 is a RPV, developed by Israel Aerospace Industries (IAI) and is equipped with electro-optical sensors for day / night observation and a laser pointer (Multi-Mission Optronic Stabilized Platform, MOSP). Its crew consists of two operators: the Air Vehicle Operator (AVO) as Pilot in Command (PIC), who is either holds a Commercial Pilot Single Engine Land including Instrument Rating as a minimum or is a licensed military pilot, and the Payload Operator (PO). The highly sophisticated autopilot system performs full automatic takeoffs and landings. The Heron 1 operating system, however, consists of additional parts besides the aircraft, such as the Advanced Ground Control Station (AGCS), the Ground Data Terminal (GDT), and the Ground Satellite Terminal (GST).

The maximum takeoff mass of a Heron 1 is $1,150 \mathrm{~kg}$, including the maximum payload mass of $250 \mathrm{~kg}$. A Rotax 914 turbocharged piston engine with 115 horsepower (HP) powers the RPV, enabling it to climb to an operational altitude of up to $30,000 \mathrm{ft}$, while its fuel capacity allows a maximum operational endurance time of 27 hours of flight time. The wingspan of the RPV measures 16,6 meters with a retractable landing gear. All control surfaces are redundant (two ailerons each wing, two rudders, and a split elevator).

Communication to and from the RPV is ensured via two redundant data links up to a line of sight (LOS) distance of $250 \mathrm{~km}$ between the GDT and the 
aircraft. In SATCOM configuration, this distance may be increased to beyond LOS. For navigation, the RPV system uses two Differential Global Positioning Systems (DGPS) for an Automatic Takeoff \& Landing (ATOL) function and two conventional Global Positioning Systems (GPS) in combination with a two Inertial Navigation Systems (INS) for enroute navigation. In the case of GPS failure, a degraded navigation using INS or distance and bearing information by the GDT is possible. In case of link loss between the RPV and the AGCS, the RPV switches to a Return Home (RH) mode. In this mode, the RPV follows a pre-programmed route (RH-Route) back to the deployment base, followed by an automatic full stop landing.

The RPV is controlled by the AVO in the Advanced Ground Control Station (AGCS), containing two redundant control bays. If one bay fails, the AVO and PO are able to immediately shift to a new control suite in the second bay. Should the entire AGCS fail, i.e. due to a fire or a loss of electrical power, which cannot be compensated by the emergency generator or batteries, RPV control may be transferred to another AGCS.

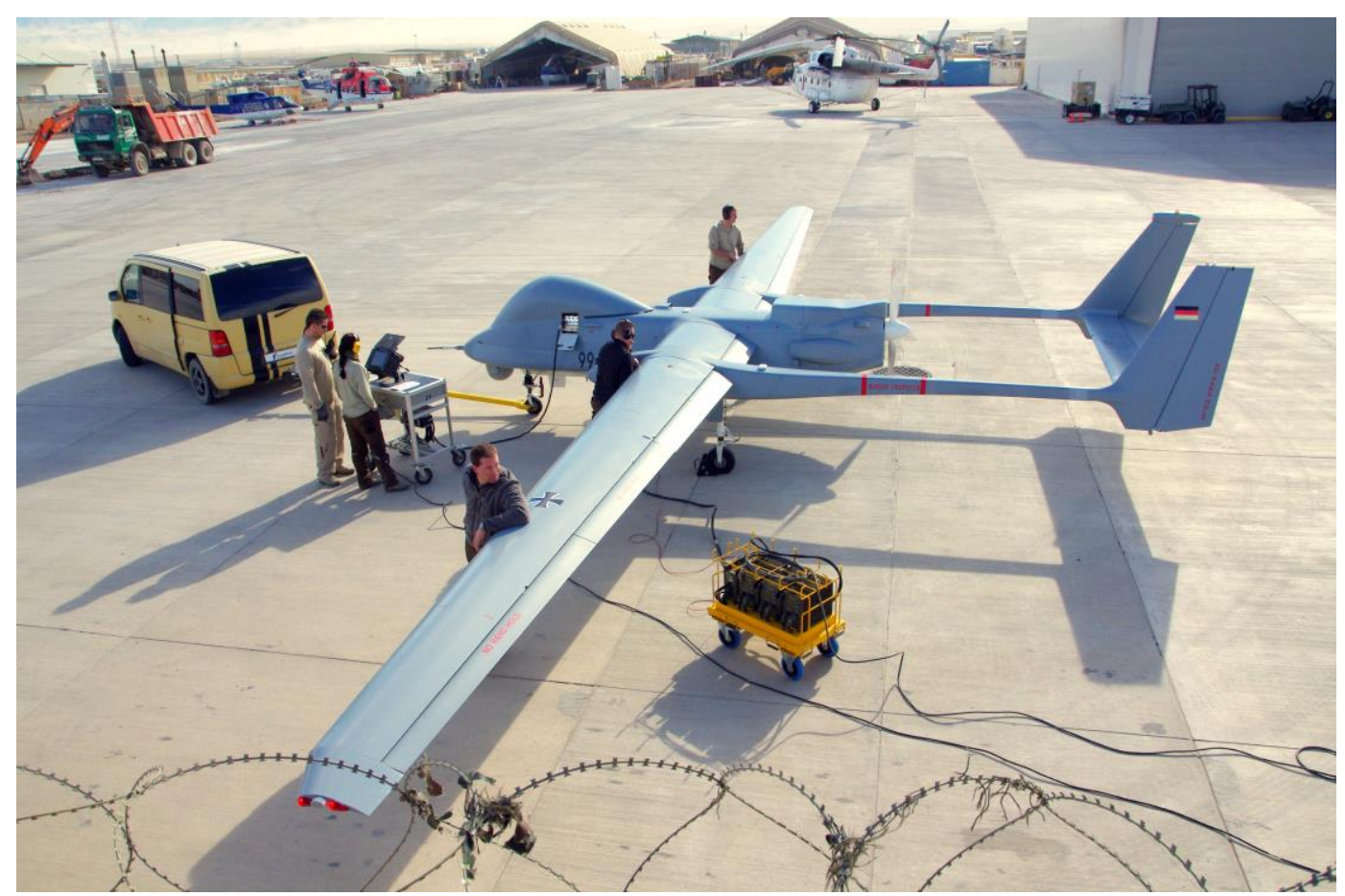

Figure 1. Heron 1 RPV. Farsch, D. (Photographer). (20 March 2011). Heron 1 [digital image].

Control inputs from the AGCS are transmitted by fiber-optic cable to a trailer mounted Ground Data Terminal (GDT) and then sent to the RPV via data link, using a directional dish and omnidirectional antenna. The directional dish antenna is able to turn 360 degrees of azimuth and to adjust its declination in relation to the RPV's position in space. 
For beyond LOS operations, a Ground Satellite Terminal (GST) is connected to the AGCS with a fiber optic cable. The GST transmits the control signals to a geostationary satellite, which in turn communicates via data link with the RPV.

\section{The Air Traffic Control System}

According to a normal RPV flight profile, different airspace classes have to be crossed to accomplish its mission. For this study, a specific airspace around Schleswig AFB, Germany will be examined (fig. 3).

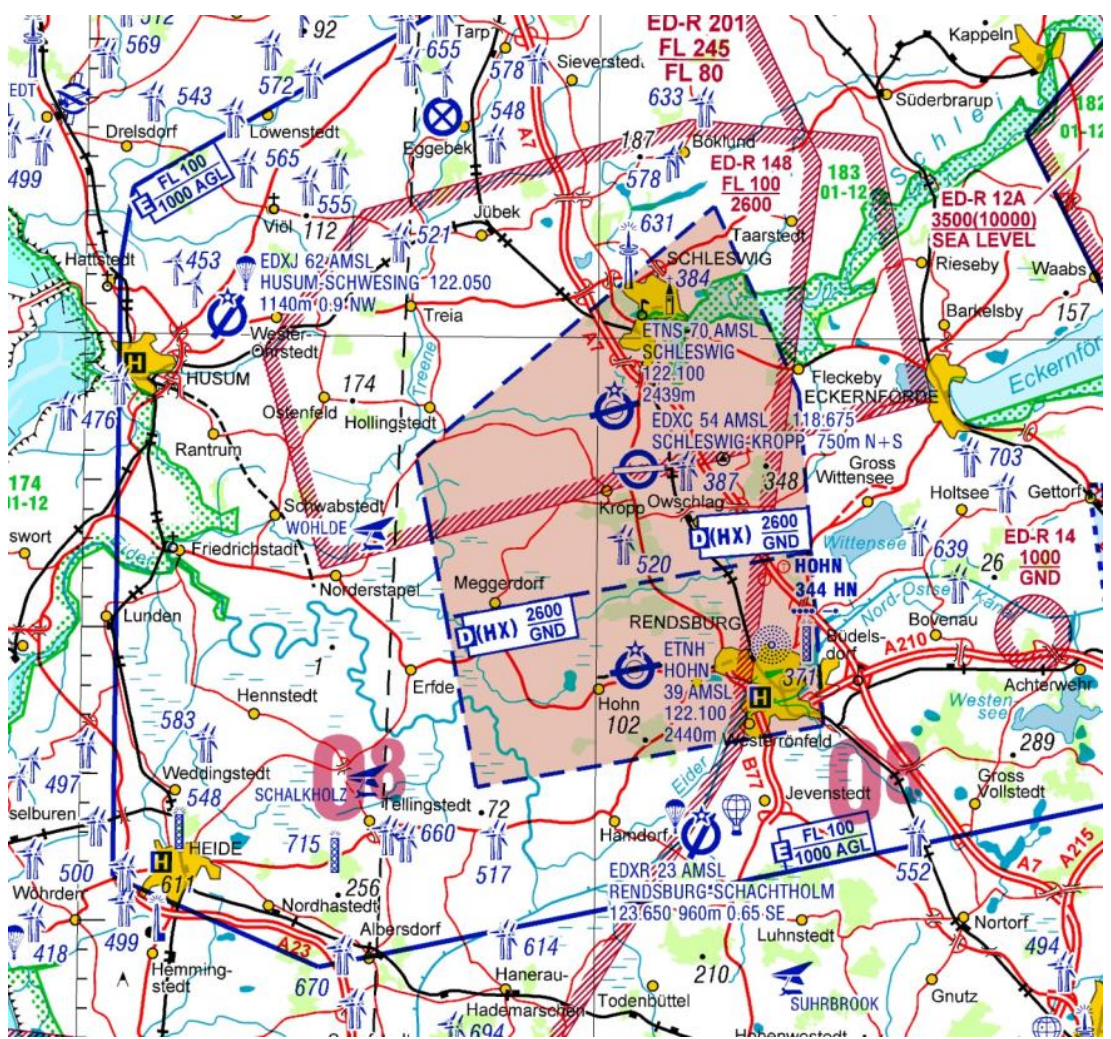

Figure 2. Airspace near Schleswig, (C) DFS Deutsche Flugsicherung GmbH (2015).

Takeoff and landing of the RPV will take place within Class D airspace. Inside class D airspaces all traffic is known to ATC, which assures separation (EU Commission, 2012).

Similarly, in the airspace above FL 100 (class C), all traffic is required to obtain an ATC clearance and separation is assured for IFR and VFR traffic by ATC. Figure 5 shows that there are two options for a RPV to reach class $\mathrm{C}$ airspace: either a circling climb in class C / D airspace above the airfield or a straight climb through class E airspace. 


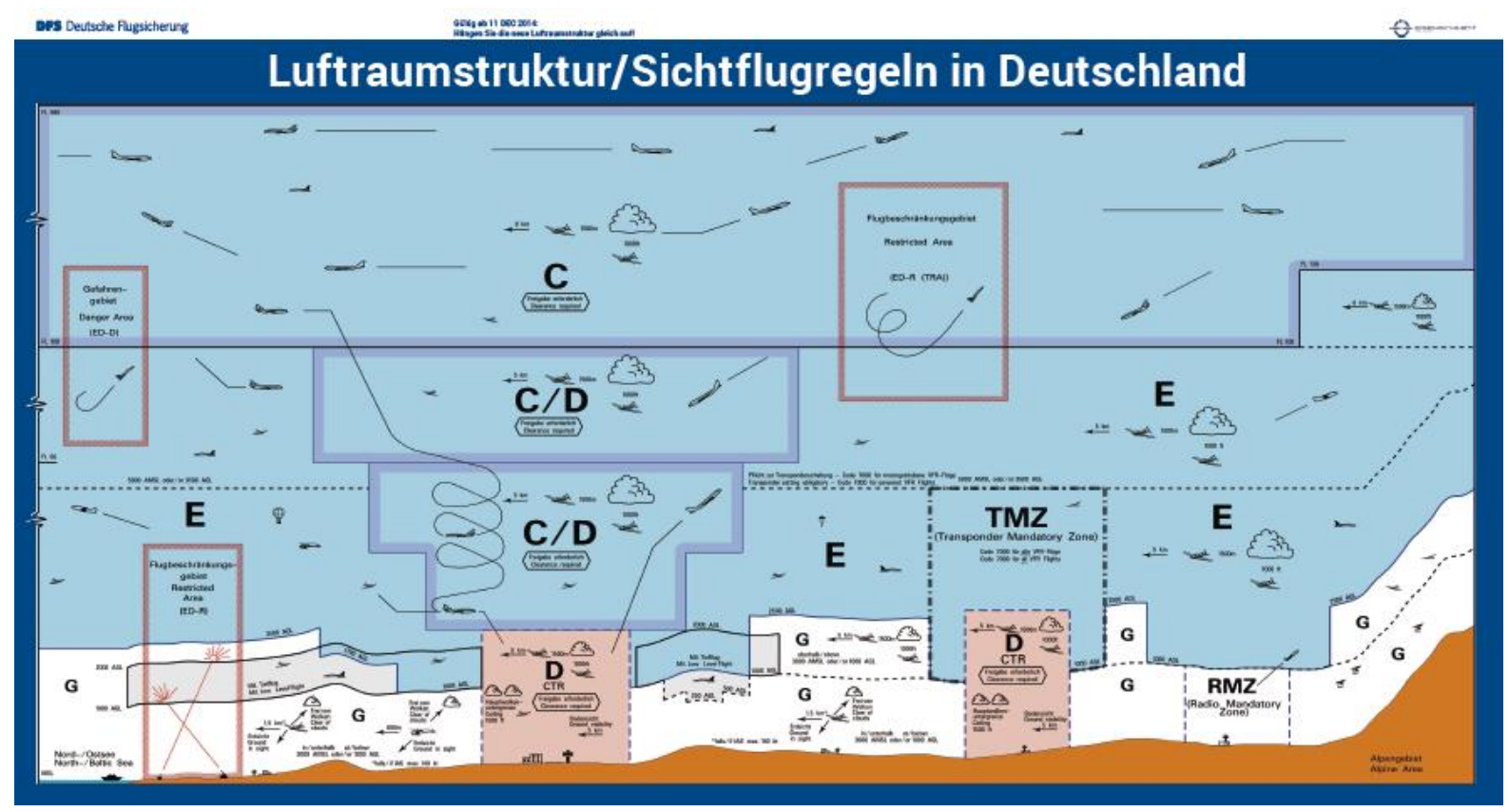

Figure 3. European Airspace Structure. (C DFS Deutsche Flugsicherung GmbH (2015).

Unless otherwise defined, no ATC clearance is required for VFR flights in class $E$ airspace. IFR flights inside class $E$ airspace are only separated from other IFR flights; separation to VFR traffic is based on the see and avoid principle.

In the unique situation of Schleswig AFB, the gap between classes D and $C$ airspace is closed by the adjacent restriced airspace (ED-R 148), established in this location to provide an effective protection against VFR flights (DFS, n. d.). Thus, under normal situations a RPV could take off from Schleswig AFB and gain altitude within class $D$ and the restricted airspace until reaching class $\mathrm{C}$ airspace - without encountering any VFR traffic.

\section{Assumptions}

Based on the RPV's system components, the present ATS, and associated regulatory framework, the following eleven specific assumptions are made:

1. It is assumed that all RPV-operators have valid licenses.

2. The RPV is at least certified as an experimental aircraft in accordance with current EASA guidelines for aircraft certification.

3. The data link to and from the RPV guarantees a safe and secure performance at all times, except in the respective failure scenarios. 
4. Jamming or spoofing the communication link to and from the RPV is impossible.

5. The European airspace enjoys uninterrupted radar coverage in the standard altitude bands, enabling a continuous, real time surveillance of the RPV while flying in that airspace.

6. The RPV is capable of prolonged operations in all-weather situations during day- and nighttime. Safe operations are possible throughout the whole atmosphere band, including every possible altitude, temperature, and humidity.

7. The navigational requirements for lateral and vertical separation are in correspondence with the EASA guidelines. Technical redundancy guarantees not only required backup systems, but also independent and sufficient energy resources.

8. Exterior lighting and marking are in accordance with EASA regulations and help to visually identify the RPV over long distances.

9. All commercial air transport aircraft, especially those operating in class $\mathrm{C}$ and $\mathrm{D}$ airspace are equipped with TCAS.

10. The imagined converging vector for the Fault Tree Analyses (FTA)s will lead to a midair collision without intervention.

11. For the taxation of likelihoods in terms of the FTA, the flight operation out of Schleswig AB, North Germany is assumed to consist of a maximum of 5 RPV flights per day.

\section{Methodology}

For the purpose of this paper, logical and graphical representations of six different scenarios depict potential sequences of events cascading into an assumed, catastrophic top event, a mid-air collision between a RPV with a conventionally piloted aircraft (Ericson, 2005). The method used to assess associated risk with this potential catastrophe is based on a modified Fault Tree Analysis (FTA), which are often used to quantify risk resulting from a potential sequence of events, leading up to a catastrophic incident (ICAO, n. d.). Moreover, an FTA "is easy to perform, easy to understand, provides useful system insight, and shows all of the possible causes for a problem under investigation" (Ericson, 2005, 183).

From the top event downward, the lower order events will be assessed regarding their maximum allowable frequency, calculated by Boolean logic and an application of Bayesian Nets. The required probability ratios to complete these calculations have been determined by an expert group during two weekend workshops.

The expert group consists of seven experienced aviators, all of which are professional pilots with military and / or civil flying background. Four experts 
have considerable work experience as flight safety officers, while five group members are educated CRM trainers and human factors specialists within their military or civil organizations. Furthermore, one participant in the expert group enjoys over four years of experience as a civil RPV pilot.

One limitation is, however, noteworthy: in order to examine all aspects of a possible midair collision between a manned aircraft and a RPV, an ATC controller would have been a desirable additional subject matter expert.

According to Deutsche Flugsicherung GmbH (DFS), the desirable safety goal for air traffic within European ATS is a maximum allowable frequency of one event in 15,000 years for a single catastrophic event within its area of responsibility to ensure an overall safety goal of one catastrophic accident per 30 years. Eurocontrol's safety goal is based on the same values (DFS, 2004, p. 1-52). From a base value of one event in 15000 years for a mid-air collision as the single event in question, top-down probability assessments are made to answer the question with what probability a lower order failure event would result in a higher order event, eventually leading up to the catastrophic mid-air collision. Out of the base value of one event in 15000 years and the probability assessments, the maximum allowable frequency of the bottom events or causes can be calculated. A more detailed description of the entire procedure will be provided in the next chapter.

\section{Results}

The following six FTA assessments depict typical failure modes, which were determined by the expert group as realistic. In every scenario, out of a normal operations condition, a failure mode becomes evident. This initial failure mode then initiates a potential sequence of events, which would eventually result in a mid-air collision between the RPV and a conventionally piloted aircraft.

It is the goal of the following FTAs to calculate the frequency of this initial failure mode and compare it to the maximum allowable frequencies of occurrence within current regulations. As a result of this comparison, the risk level of of RPV integration in the current ATS may be determined and, ultimately, risk mitigation measures derived. 


\section{An aircraft violates minimum separation criteria to the RPV in} airspace C, D, ED-R

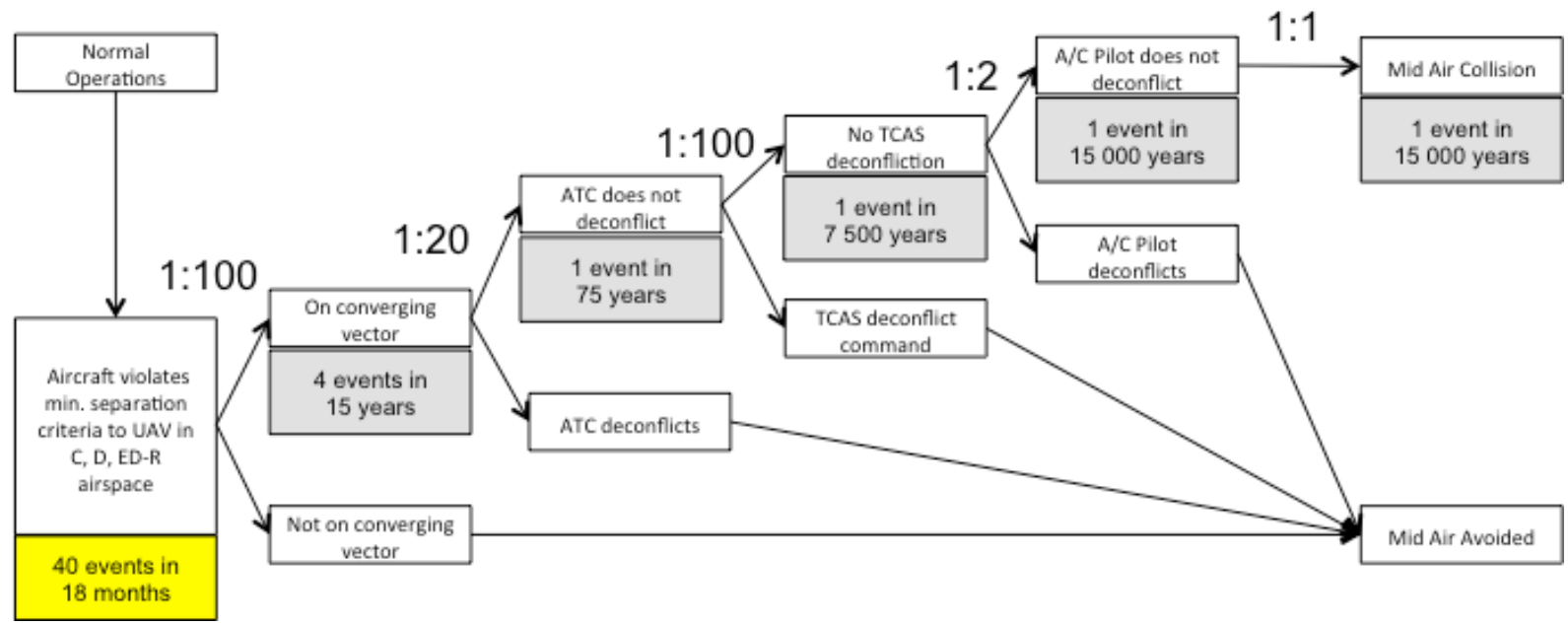

Figure 4. Violation of minimum separation criteria to RPV in controlled airspace

Fault Tree. Out of normal air traffic operations on any given day, in this scenario an aircraft violates minimum separation criteria to the RPV (ICAO, Doc 4444 ATM/501, 2007). Reasons for the aircraft violating the airspace could be manifold, including ATC errors, navigational problems (e.g. pilot error, NAV database error, weather deviations), flight path deviation due to an inflight emergency (e.g. emergency decent) or other manifestations of human error, equipment failure, or environmental causes such as a weather deviation VFR traffic penetrating the airspace without clearance is included in this scenario.

If the RPV and the other air traffic are not on a converging vector, a midair collision will not occur. Conversely, if both aircraft are on converging vectors, a midair collision would be the result - under the assumption that every other safety barrier fails. In the case of this and every following scenario, ATC would function as the next layer of defense. Should ATC deconflict in time, a midair collision will be avoided. If, however, ATC does not deconflict, the next safety layer will depend on the functionality of the Traffic Collision Avoidance System (TCAS). For TCAS-equipped aircraft, there is a high likelihood that TCAS commands will prevent a collision with the RPV. For non-TCAS aircraft - or in the case of TCAS commands not being followed - the next safety barrier solely depends on a "see and avoid" maneuver by the pilot of the conflicting aircraft. 
Taxation of Likelihoods. The likelihood of each lower order event causing a higher order effect will be assessed in a top-down approach by the expert group conducting this study. In this scenario, the probability that a midair collision occurs after every barrier has failed is $100 \%$ - under the assumption that the aircraft pilot does not "see and avoid" the RPV at the last moment. It is further assumed that the visual identification technology available to the AVO is not being used for "see and avoid" purposes. In case that a pilot does not follow a TCAS advisory, the probability that the pilot will visually acquire the RPV and, thus, be able to avoid a collision at the last moment has been assessed as $50 \%$. The probability, in turn, that TCAS will not advise a deconflicting flight path beforehand is judged as one percent (also assuming that not every aircraft in $\mathrm{C}, \mathrm{D}$, and ED-R airspace is TCAS equipped), whereas the probability that ATC does not correct a dangerous situation resulting from a converging flight path is taxed at 5\%. The likelihood of a RPV and an aircraft initially being in the same airspace at the same time as a result of a systemic failure in air traffic is assessed as 1:100.

Risk. ESARR 4 (Safety Regulation Commission, 2001) introduces the following risk matrix for European ATC's area of responsibility:

Table 1

\begin{tabular}{|l|l|l|l|l|l|}
\hline \multirow{2}{*}{$\begin{array}{l}\text { Occurrence of the } \\
\text { effect } \\
\text { (Once per...) }\end{array}$} & \multicolumn{5}{|l|}{ Severity of Effect } \\
\cline { 2 - 7 } & Catastrophic & Large & Medium & Low & None \\
\hline$<1$ hour & a & a & a & b & d \\
\hline 1 hour .. 5 days & a & a & b & c & d \\
\hline $\begin{array}{l}5 \text { days ... 18 } \\
\text { months }\end{array}$ & a & b & c & d & d \\
\hline $\begin{array}{l}18 \text { months ... 150 } \\
\text { years }\end{array}$ & b & c & d & d & d \\
\hline $\begin{array}{l}150 \text { years ... 15 } \\
000 \text { years }\end{array}$ & c & d & d & d & d \\
\hline$>15000$ years & d & d & d & d & D \\
\hline
\end{tabular}


Risks classified as "a" and "b" shall not be accepted. Risks classified as "c" are acceptable under certain conditions, such as a temporary acceptance. In any case, risk mitigation actions should be considered to lower these risks. Risks classified as "d" do not need further attention in the view of ESARR 4.

Similar to risk classification, the safety goals for the occurrence of the initial event will be calculated from the maximum occurrence rate times the probability of its effect, too. The resulting safety goal criticality will be determined according to the following table (DFS, 2004).

Table 2

\begin{tabular}{|l|l|}
\hline Severity of Effect & Maximum Occurrence Rate \\
\hline Catastrophic & Once in 15000 years \\
\hline Large & Once in 150 years \\
\hline Medium & Once in 18 months \\
\hline Low & Once in 5 days \\
\hline
\end{tabular}

The overall safety goal for a midair collision between the RPV and an aircraft is once in 15000 years. Considering that every safety barrier fails with certain probabilities from the start of the chain of events until a potential midair collision, the safety goal for an aircraft violating minimum separation criteria to a RPV in airspace C, D, and ED-R is of 40 times in 18 months. This number indicates the system's resilience, indicating that the overall safety goal will not be jeopardized, even if an aircraft violates minimum separation criteria in airspace C, D, ED-R up to 40 times in 18 months. Overall, the criticality of this failure mode is medium. 


\section{RPV deviates from cleared route in C, D, ED-R airspace}



Figure 5. RPV deviates from clearance in controlled airspace

Fault Tree. This scenario describes the event of the RPV deviating from its intended and cleared route in C, D, ED-R airspace. It is assumed that besides technical failures, human factor issues will mainly cause these deviations. Operator errors may include unintended RPV flight modes, a (pre-) selection of incorrect navigation points, or the utilization of unfitting NAV databases.

Taxation of Likelihoods. Compared to the likelihood values of the previous scenario, this scenario differs only in one case. If the RPV deviates from cleared route in $\mathrm{C}, \mathrm{D}, \mathrm{ED}-\mathrm{R}$ airspace, the probability to end up on a converging vector in relation to another aircraft is assessed at 1:20 due to the volume of air traffic surrounding the RPV in non-segregated airspace.

Risk. As a result of the differing probabilities in this scenario, the safety goal for this failure mode is reduced to 8 events in 18 months. Nevertheless, the overall criticality of a RPV deviating from its cleared route in C, D, or ED-R airspace remains medium. 
3. RPV deviates from cleared route and one aircraft with no communication link to ATC in same airspace

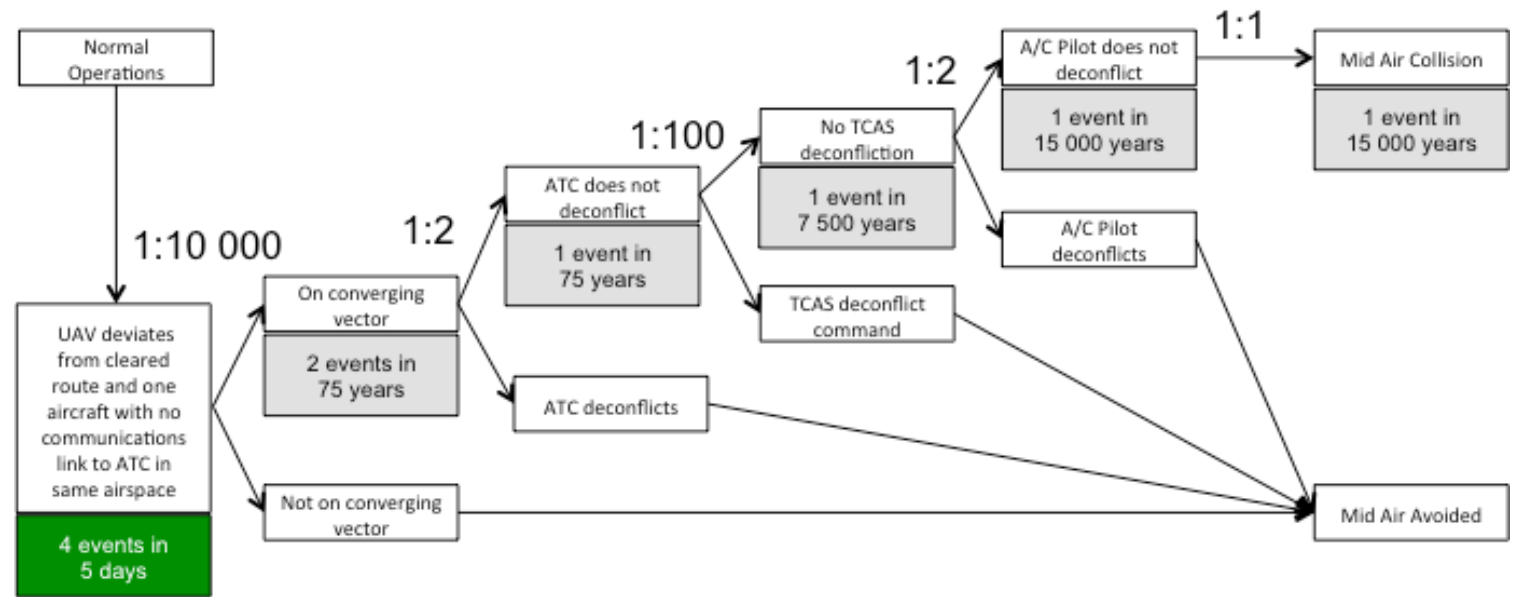

Figure 6. RPV deviates from clearance without communication link to ATC

Fault Tree. In this scenario, a RPV deviates from its cleared flight path, while another aircraft is in the same airspace, at the same time, without communication to ATC. The RPV's deviation could result from technical reasons (e.g. lost data link, engine failure, and other catastrophic failures) or simply due to operator error. The conflicting aircraft could have encountered a lost comm situation due to technical malfunctions or due to operator error, (e.g. switching to a wrong frequency). This scenario describes a critical case, in which a RPV may act unpredictably or with impaired maneuverability, while ATC cannot provide the lost comm aircraft with avoidance vectors.

Taxation of Likelihoods. The risk assessment of this scenario deviates from the first case at the first two safety layers. First, the probability a RPV deviating from its cleared flight path and ending up on a converging vector with another aircraft suffering lost comm with ATC is assessed as one in 10000 . Second, if it does happen, nonetheless, the likelihood of ATC being unable to issue avoidance vectors will increase to $50 \%$. This is mainly due to the reduced number of options left to ATC, which depend on the cause for the flight path deviation by the RPV.

Risk. Mainly due to the low probability of both failure modes occurring on a converging flight path, the safety system will theoretically tolerate 4 RPV deviations from a cleared route and an aircraft with no radio connection with ATC in the same airspace in 5 days. Hence, the overall criticality of this failure mode is low. 


\section{Loss of RPV surveillance feed to ATC}

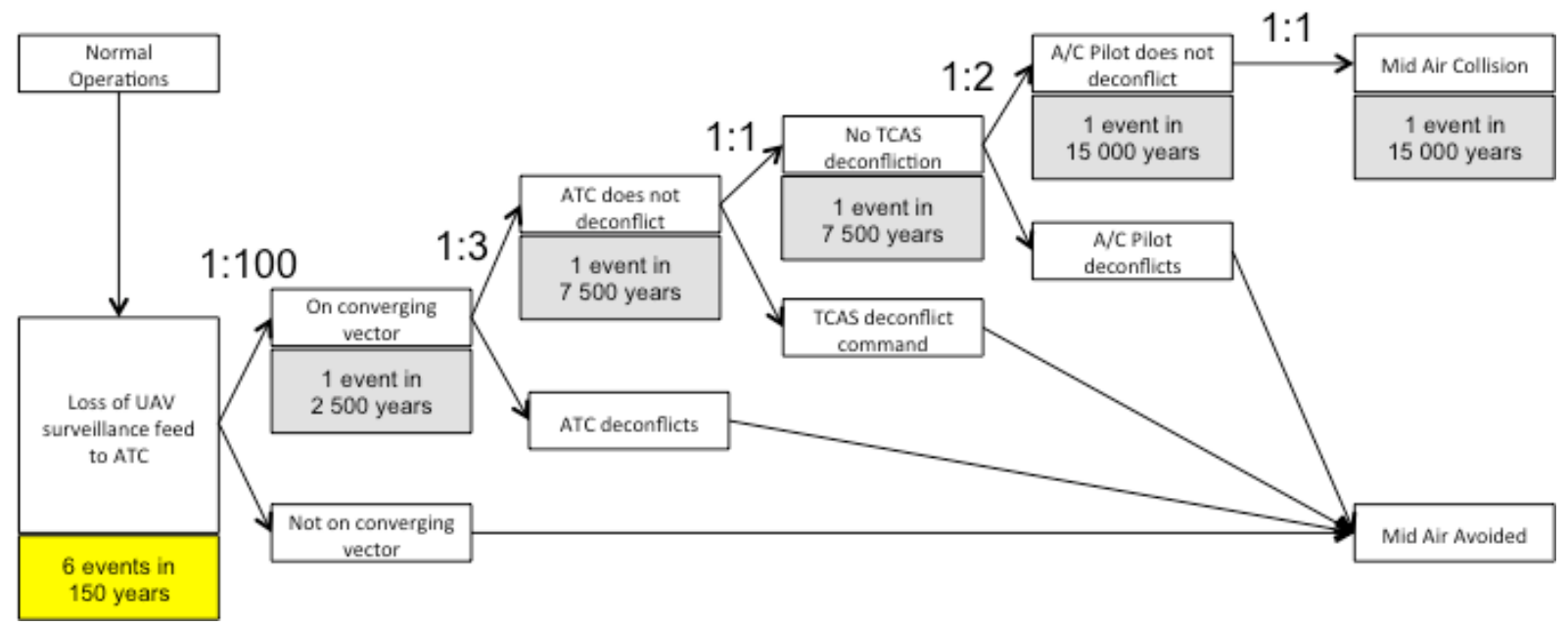

Figure 7. Loss of RPV surveillance feed to ATC

Fault Tree. Secondary Surveillance Radars (SSR) interrogate transponders of airborne platforms, thereby providing relevant information on the target's transponder squawk and flight level. Based on these parameters, ATC provides traffic deconfliction. In this scenario, ATC loses the RPV's transponder signal due to a technical malfunction in the RPV system.

Taxation of Likelihoods. In this scenario, the possibility that ATC will not revert to primary radar based tracking methods and therefore fail to deconflict a RPV on a converging vector toward another aircraft is judged to be one in three cases. Moreover, due to an absent transponder signal, the RPV will not trigger TCAS alerts of other aircraft on a converging vector, thus increasing the likelihood of a missed TCAS deconfliction to $100 \%$.

Risk. A lost transponder signal is a problematic case in aviation controlled by secondary radar, regardless of the affected aircraft being manned or unmanned. All probability assessments included, the system will tolerate 6 lost transponder signals in 150 years to meet the overall safety goal of one midair collision in 15000 years with a medium criticality. 


\section{Loss of surveillance feed to ATC plus Comm Loss between RPV operator and ATC}

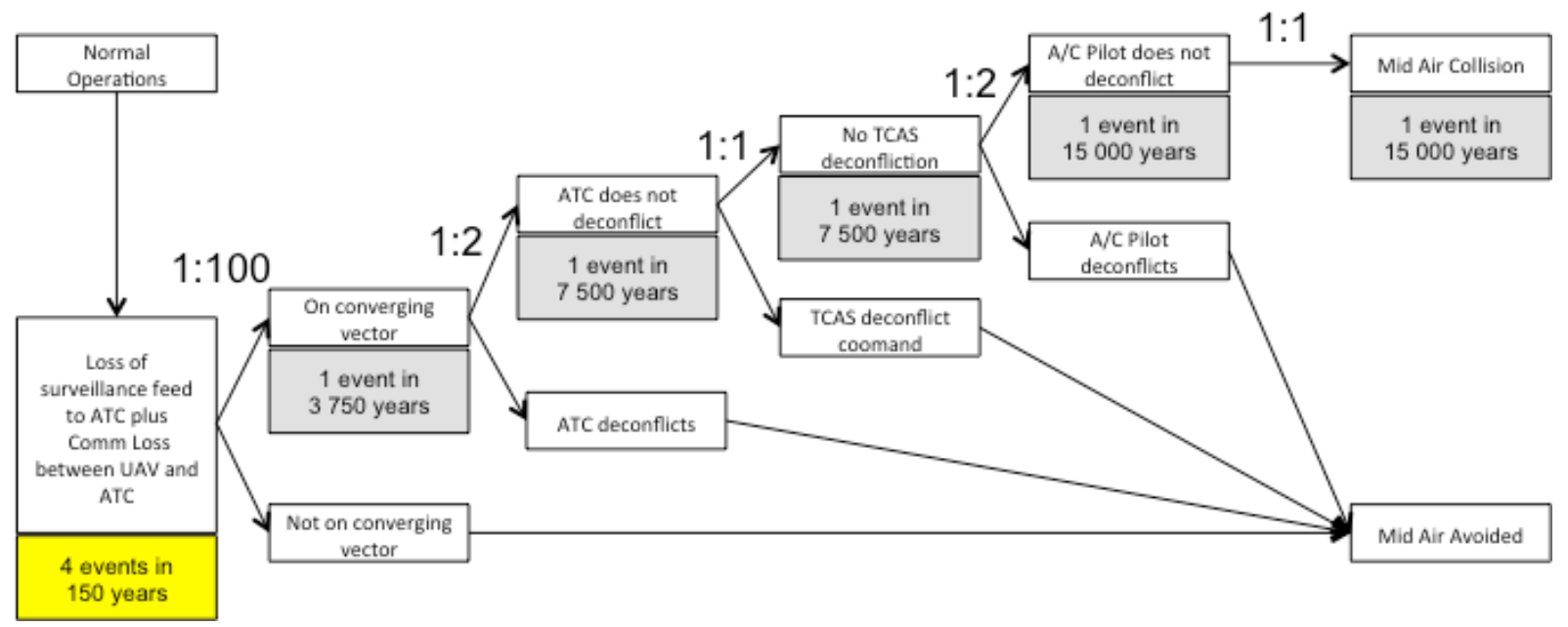

Figure 7. Loss of RPV surveillance feed to ATC

Fault Tree. In this scenario, ATC loses the RPV's transponder signal, while simultaneously losing radio contact to the RPV controller.

Taxation of Likelihoods. The expert group considers the simultaneous occurrence of this case and a resulting flight path conflict as extremely low, namely $1 \%$ only. However, in contrast to the previous case, the likelihood that ATC will not deconflict a RPV on a converging vector with another aircraft is increased significantly to $50 \%$ due to the reduced number of options still available to ATC.

Risk. Again, the overall safety goal for this case is once in 15000 years. Using the previously mentioned calculations, the safety goal is 4 events in 150 years with medium criticality. 


\section{RPV deviates into $\mathrm{E}$ airspace}

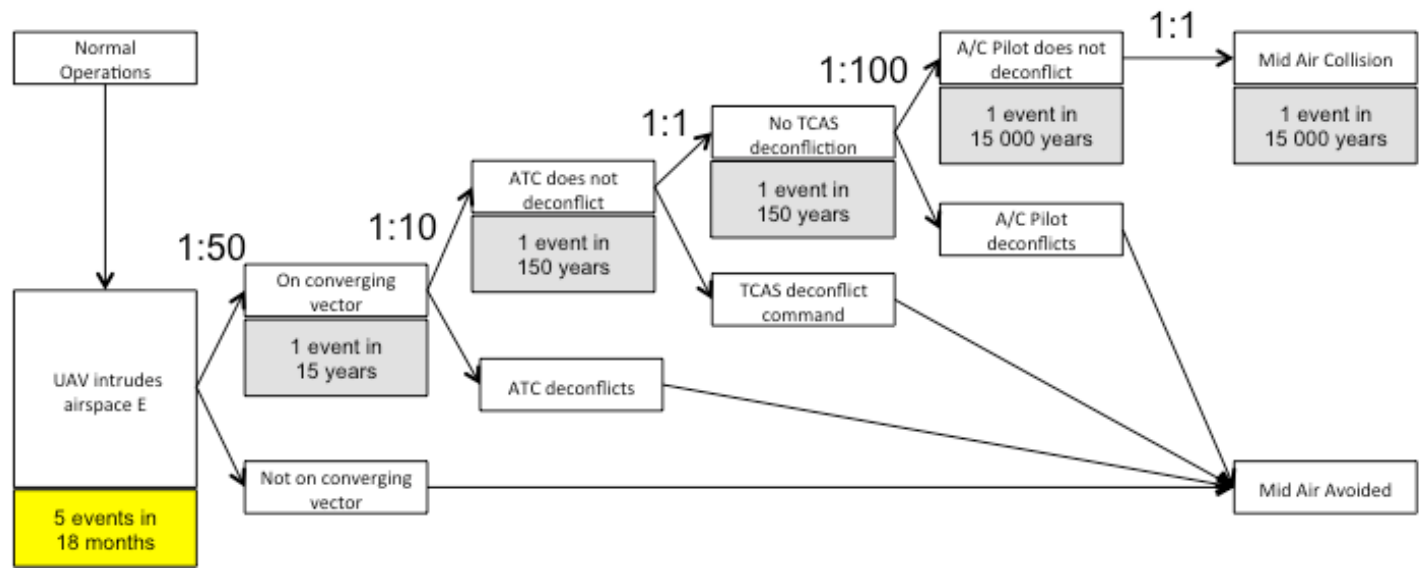

Figure 8. RPV deviates into E airspace

Fault Tree. In this scenario, the RPV enters airspace E unintentionally or due to an inflight emergency, possibly leading to separation conflicts with other controlled or uncontrolled air traffic. Possible causes include ATC errors, navigational errors by the RPV operator, RPV navigation equipment failures (e.g. navigation database error, navigation sensor failure, altitude sensor failure) or other technical problems (e.g. engine failure, stuck rudder). In case of a RH scenario, the RH route could also penetrate airspace $\mathrm{E}$ due to an initial false programming.

Taxation of Likelihoods. The likelihood of a converging vector in this scenario is 1:50 - under the assumption that a comparably higher number of VFR and IFR traffic uses airspace $E$ at the time of the event. Due to the fact that only a certain portion of these aircraft will be under ATC control, the likelihood of a missed deconfliction by ATC is considerably higher. Similarly, most VFR aircraft in this airspace will not be equipped with TCAS; hence the likelihood of a TCAS deconfliction is reduced to $5 \%$. On the other hand, VFR pilots normally proceed according to the principle of "see and avoid". Thus, it is assumed that the probability of a successful deconfliction by a converging pilot is $100 \%$.

Risk. To maintain the overall safety goal of 1 event in 15000 years, the safety goal for RPV intruding airspace $\mathrm{E}$, for one of the previously mentioned reasons, equals 5 events in 18 months. 


\section{Discussion}

If an aircraft violates minimum separation criteria in airspace C, D, ED$\mathrm{R}$, a higher level of safety could be achieved by issuing the RPV pilot with a Recognized Air Picture (RAP), displaying secondary radar returns in the vicinity of the RPV. By using a RAP, the probability of a RPV-operator induced deconfliction after ATC and TCAS have failed to do so, could be increased. In addition, equipping the RPV with TCAS, thus potentially advising a deconflicting flight path to the RPV-operator, would further decrease the overall risk.

If the RPV deviates from cleared route in C, D, ED-R airspace, a RAPequipped RPV-operator in combination with TCAS would similarly reduce the associated risks. That same risk mitigation technique would work in case that the RPV deviates from its cleared route and one aircraft with no communication link to ATC is in same airspace. Both readily available measures would provide an additional layer of safety.

In case that the ATC loses its surveillance feed from the RPV, a RAP would only then increase the overall level of safety, if an alternative means of determining the RPV's exact location, altitude, and flight path by means of an alternate data link between the RPV and its ground station is available to the $\mathrm{RPV}$-operator. If this is indeed the case, the RPV operator could provide flight path deconfliction if needed as an additional safety measure. However, further examination would be needed to determine the exact probabilities and frequencies of safety gain to justify the necessary investments in technology.

Similarly, if ATC loses its surveillance feed from the RPV plus the radio contact has been lost between the RPV-operator and ATC, a RAP would only then significantly increase the level of safety, if the RPV's position can be tracked and displayed by alternative means. A more promising approach to mitigate risk in this case would be to strive for additional technical redundancy. A simple quick-dial telephone connection from ATC-controller to RPVoperator could be one such means. In the remote case of a simultaneous radio communications to the RPV and transponder signal-loss, ATC-controllers and RPV-operators would be able to coordinate the RPV's flight path and together decide on the best deconfliction strategies.

Finally, if a RPV deviates into E airspace, an autonomously initiated transponder setting of 7700 should be mandatory upon the recognition of a flight path deviation. In case of a transponder failure on the RPV's side, intense communication between ATC and the RPV-operator is necessary to report the actual position and altitude of the RPV on ATC request. Similar to the other scenarios, a RAP-equipped RPV-operator would further reduce the risk of a midair collision. Equipping the RPV with TCAS potentially advising a deconflicting flight path to the RPV-operator, would further decrease the overall 
risk of RPV integration into the ATS. Restricting the RPV to night missions would eliminate the threat of encountering random VFR traffic, since at night, all VFR flights are must be in radio contact with ATC.

\section{Conclusion}

This study aimed to examine whether RPV integration into the existing ATS and within the current regulatory framework would be safe enough today - without the availability of commercial off-the-shelf sense and avoid technology. Yet, it was not the goal of this study to provide a legal argument for the legal air traffic integration of RPVs without this technology.

Results indicate that even without any risk mitigation changes, the maximum risk level would not exceed a medium risk to overall flight safety. With an implementation of four simple risk reduction measures, a significant reduction of risk would be possible. These measures are:

- Equip the RPV-operator with a real time recognized air picture, thus enabling the operator to gain and maintain situational awareness on the traffic situation in the vicinity of the RPV.

- Equip the RPV with TCAS, thus providing one additional layer of safety for the purpose of preventing a mid-air collision with other TCAS equipped aircraft.

- Install a quick-dial land line phone connection between the RPVoperator and Air Traffic Control. This would provide an additional, effective, and RPV-exclusive means of communication in the event of radio communication failures between ATC and the RPVoperator.

- Start the integration of RPVs into the ATS at night. While the safety level of see and avoid may be reduced in darkness, the fact that all air traffic - even in airspace E must establish two-way radio contact to ATC at night, will actually reduce the overall risk of a mid-air collision between a RPV and a conventionally piloted aircraft in this type of airspace.

In summary and in the view of the authors of this study, RPVs may be integrated into the current ATS with a tolerable risk to air traffic, if these four recommendations are followed. Yet it is impossible to calculate whether an implementation of these recommended risk reduction measures would actually result in a low risk in every one of the scenarios above. Thus, further practical simulation and local implementation studies are needed to, first, confirm the theoretical results of this study and second, to investigate whether these risk reduction measures have an actual impact on occurrence frequencies or on the probabilities of higher order events resulting from lower order occurrences. 


\section{Abbreviations}

$\begin{array}{ll}\text { A/C } & \text { Aircraft } \\ \text { AGCS } & \text { Advanced Ground Control Station } \\ \text { ATOL } & \text { Automatic Takeoff \& Landing } \\ \text { ATS } & \text { Air Traffic System } \\ \text { AVO } & \text { Air Vehicle Operator } \\ \text { DGPS } & \text { Differential Global Positioning System } \\ \text { GAF } & \text { German Air Force } \\ \text { GDT } & \text { Ground Data Terminal } \\ \text { GPS } & \text { Global Positioning System } \\ \text { GST } & \text { Ground Satellite Terminal } \\ \text { HP } & \text { Horsepower } \\ \text { IAI } & \text { Israel Aerospace Industries } \\ \text { INS } & \text { Inertial Navigation System } \\ \text { IR } & \text { Infrared } \\ \text { MALE } & \text { Medium Altitude Long Endurance } \\ \text { MOSP } & \text { Multi-Mission Optronic Stabilized Platform } \\ \text { PIC } & \text { Pilot in Command } \\ \text { PO } & \text { Payload Operator } \\ \text { RAP } & \text { Recognized Air Picture } \\ \text { RH } & \text { Return Home } \\ \text { RPV } & \text { Remotely Piloted Vehicle } \\ \text { SA } & \text { Situational Awareness }\end{array}$




\section{References}

BBC News. (2013, December 2). Amazon testing drones for deliveries. Retrieved from http://www.bbc.com/news/technology-25180906

Bill, C. (2015, January 6). ICAO panel will recommend first UAV standards in 2008. AINonline. Retrieved from http://www.ainonline.com/aviation-news/aerospace/2015-01-06/icaopanel-will-recommend-first-uav-standards-2018

DFS Deutsche Flugsicherung GmbH. (n.d.). Standardized European Rules of the Air (SERA). Luftraumstruktur/Sichtflugregeln in Deutschland gültig ab 11.12.2014 Retrieved from http://www.dfs.de/dfs_homepage/de/Flugsicherung/ Luftraum/Luftraumstruktur_11122014.pdf

DFS Deutsche Flugsicherung GmbH. (2014). Aeronautical Chart ICAO 1:50000. Hamburg (NO53/6). Edition 2014. Langen, Germany: Author.

DFS Deutsche Flugsicherung GmbH. (2004). Handbuch der Sicherheitsbewertungen. Langen, Germany: Author.

DFS Deutsche Flugsicherung GmbH. (2006). Handbook Safety Assessment Volume 1. Introduction into Methodology. Langen, Germany: Author.

Ericcson, C. A. II. (2005). Hazard Analysis Techniques for System Safety. Hoboken, New Jersey: John Wiley \& Sons, Inc.

European Commission. (2012). Commission Implementing Regulation (EU) No 923/2012 of 26 September 2012 laying down the common rules of the air and operational provisions regarding services and procedures in air navigation and amending Implementing Regulation (EU) No 1035/2011 and Regulations (EC) No 1265/2007, (EC) No 1794/2006, (EC) No 730/2006, (EC) No 1033/2006 and (EU) No 255/2010 Text with EEA relevance. Official Journal of the European Union. Retrieved from http://eur-lex.europa.eu/legalcontent/EN/TXT/?qid=1431679073336\&uri=CELEX:32012R0923

EUROCONTROL. (2009). Safety Regulation Commission Document 12. Assessment of the EATM 'Air Navigation System Safety Assessment Methodology' as Means of Compliance with ESARR 4 ( $\left.2^{\text {nd }} \mathrm{ed}.\right)$. Retrieved from http://www.eurocontrol.int/articles/safety-assessment-methodologysam 
Federal Aviation Administration. (n.d.). Unmanned Aircraft Systems (UAS) Regulations \& Policies. Retrieved from https://www.faa.gov/uas/regulations_policies/

International Civil Aviation Organisation. (n.d.). Fault tree analysis and event tree analysis [Brochure]. Retrieved from http://www.icao.int/SAM/Documents/2014ADSAFASS/Fault\%20Tree\%20Analysis\%20and\%20Event $\% 20$ Tree\% 20Analysis.pdf

International Civil Aviation Organisation. (2011). Unmanned Aircraft Systems (UAS) Circular (Cir 328 AN/190). Retrieved from http://www.icao.int/Meetings/UAS/Documents/Circular\%20328_en. pdf

Israel Aerospace Industries. (2009). Heron system operational manual. System description manual [TM(CA)HR-001]. Tel Aviv, Israel: Author. 